\title{
Clinicopathological features of cystic lesions in the eyelid
}

\author{
YUKA SUIMON $^{1}$, SATORU KASE $^{1}$, KAN ISHIJIMA $^{1}$, HIROMI KANNO-OKADA $^{2}$ and SUSUMU ISHIDA ${ }^{1}$ \\ ${ }^{1}$ Department of Ophthalmology, Faculty of Medicine and Graduate School of Medicine, Hokkaido University, Sapporo, \\ Hokkaido 060-8638; ${ }^{2}$ Department of Surgical Pathology, Hokkaido University Hospital, Sapporo, Hokkaido 060-8648, Japan
}

Received October 19, 2018; Accepted December 4, 2018

DOI: $10.3892 /$ br.2018.1177

\begin{abstract}
The aim of the present study was to investigate the clinicopathological features of cystic lesions in the eyelid. Clinicopathological profiles were retrospectively searched based on medical records of 35 patients with cystic lesions of the eyelids, which were surgically resected from January 2003 to June 2016. The cohort consisted of 16 men and 19 women. The mean age of the patients was $57 \pm 24$ years. The main locations of the cysts were the upper eyelid in 22 patients, followed by the lower eyelid in 5 patients. Eyelid skin and its appendages were the most common as the origin of cysts, followed by the tarsus, palpebral conjunctiva and lacrimal gland. The histopathological diagnoses were 16 epidermal cysts, 5 intratarsal keratinous cysts, 3 conjunctival cysts, 2 trichilemmal cysts, 1 dermoid cyst, 1 apocrine hidrocystoma, 1 lacrimal gland cyst and 6 unclassifiable cysts. All cysts did not recur following resection. In summary, cystic lesions of the eyelid were frequently observed in the upper eyelid. The most frequent diagnoses were epidermal cysts, followed by intratarsal keratinous cysts, while a definitive diagnosis in some cysts could not be pathologically determined, as they lacked epithelia and/or the contents or they resembled apocrine hidrocystoma and intratarsal keratinous cysts with atypical findings. The results of the current study indicate that the incidence and differential diagnosis of eyelid cystic lesions may contribute to the application of appropriate treatment for patients with eyelid tumors.
\end{abstract}

\section{Introduction}

The eyelid contains various histological components including eyelid skin squamous epithelium, subcutaneous tissue (comprising eccrine and apocrine glands), the tarsal plate (consisting of meibomian glands) and palpebral conjunctiva. Cystic lesions are a type of benign eyelid tumor arising in the subcutaneous tissues of the eyelid, the tarsus and beneath the

Correspondence to: Dr Satoru Kase, Department of Ophthalmology, Faculty of Medicine and Graduate School of Medicine, Hokkaido University, Kita-ku, Sapporo, Hokkaido 060-8638, Japan

E-mail: kaseron@med.hokudai.ac.jp

Key words: eyelid cyst, epidermal cyst, intratarsal keratinous cyst, pathological diagnosis palpebral conjunctiva (1). Common eyelid cystic lesions may be characterized as epidermal or intratarsal keratinous cysts (IKC), eccrine hidrocystoma, milia or steatocystoma (1-3). The pathogenesis of eyelid cystic lesions may be associated with the occlusion of ducts within the tarsal plate or with the aberrant invagination of the epithelium $(1,4)$. Chalazion is also a common inflammatory disease arising in the eyelid, which histologically presents with lipogranuloma without cyst formation. Although cystic lesions are benign, they can be clinically confused with malignant tumors such as sebaceous carcinoma, which possibly manifest clinical features including blepharitis or chalazion (5). IKC arising in the meibomian gland has been described (1). IKC is a non-inflammatory intratarsal cyst which is considered to arise from the meibomian gland duct (1). It is important to understand the clinical features of IKC, and differentiate IKC from other eyelid diseases, as IKC requires a total excision with partial tarsectomy. Otherwise, IKC can easily relapse if removed by simple incision and/or curettage $(1,2)$. Indeed, cases of IKC have been frequently misdiagnosed as chalazia or epidermal cysts, and recurred following incomplete resection of the cysts' wall and/or curettage $(1,2)$. The incidence of IKC among cystic lesions of the eyelid, however, remains unknown. If the incidence and clinicopathological features are clearly provided, these results may contribute to the better treatment of eyelid cystic lesions and tumors. The aim of the present study was to investigate the clinicopathological features of cystic lesions in the eyelid including IKC.

\section{Materials and methods}

Patients. The present study was a retrospective observational study. The Institutional Review Board (IRB) of Hokkaido University Hospital (Sapporo, Japan) for clinical research approved the study (IRB approval no. 016-0426). Written informed consent was collected for the current study. If this was not provided, information regarding the present study was disclosed on the university website for opt out of approval. Written informed consent for the publication of the current study was gained from 5 patients, and the other 30 patients were included in the opt out of approval. The research adhered to the tenets of the Declaration of Helsinki. Patients, who underwent surgical excision of the eyelid tumors in Hokkaido University Hospital from January 2003 to June 2016, were consecutively enrolled in the study. All the excised tissues were submitted for pathological evaluations, and were diagnosed as cystic 
lesions. All cysts including the cyst walls were completely excised during surgery. Cystic lesions of the orbit, the caruncle and the bulbar conjunctiva, and solid tumors including inflammatory diseases such as chalazion were excluded from study. Furthermore, patients without informative pathological data were also excluded. The study analyzed the clinical pictures and histological findings obtained from the medical records, which were stored in the pathology laboratory of the Department of Medical information in Hokkaido University hospital (Sapporo, Japan).

\section{Results}

Clinical features. A total of 35 patients with eyelid cysts were enrolled. Table I summarizes the clinical features of the patients. The mean age was $57 \pm 24$ years (3-87 years). The patient cohort consisted of 16 men and 19 women. Cysts were observed in the right and left sides in 19 and 14 patients, respectively. However, the current study could not confirm the actual site of the cyst in 2 patients, as the respective information was absent from their records. The location of cysts was the upper eyelid in 22 patients, the lower eyelid in 5 patients, the medial canthus in 5 patients, and unknown in 3 patients. The mean follow-up period was $44 \pm 37$ months (1-159 months). All cysts did not recur during the follow-up periods.

Summary of pathological diagnoses. The origins and the final diagnoses of the cystic lesions are listed in Table I. The origins of the cysts were classified as eyelid skin and its appendages, tarsus, palpebral conjunctiva and lacrimal grand. Eyelid skin and its appendages were the most common origin of cysts $(n=20)$, followed by the tarsus $(n=5)$, palpebral conjunctiva $(n=3)$ and lacrimal gland $(n=1)$. The histopathological diagnoses were 16 epidermal cysts $(46 \%), 5 \mathrm{IKCs}$ (14\%), 3 conjunctival cysts $(9 \%), 2$ trichilemmal cysts $(6 \%)$, 1 dermoid cyst (3\%), 1 apocrine hidrocystoma (3\%) and 1 lacrimal gland cyst (3\%). Meanwhile, 6 cysts were not histologically determined.

\section{Clinicopathological features of representative eyelid cysts} Epidermal cyst. In the current study, the mean age of 16 patients with epidermal cysts was $55 \pm 28$ years. Among the 16 patients with epidermal cysts, 9 cysts were located in the upper eyelid (Fig. 1A), 4 cysts in the medial canthus and 2 cysts in the lower eyelid. The epidermal cysts exhibited walls with keratinized stratified squamous epithelia containing granular layers. The content of the cyst was laminated layers of keratin (Fig. 1B and C).

$I K C$. The mean age of the 5 patients with IKCs was $68 \pm 14$ years without obvious gender predilection (two males and three females). All lesions involved the upper eyelid presenting with a single cyst (Fig. 2A and B). Histologically, the cyst wall contained undulating stratified squamous epithelium without a granular layer (Fig. 2C and D). The content of the cyst was string-like keratin.

Other cysts. All other cysts were conjunctival cysts (3 patients), trichilemmal cysts (2 patients), dermoid cyst, apocrine hidrocystoma and lacrimal gland cyst (1 patient with each; not shown). The conjunctival cysts were lined by columnar or squamous epithelium intermixed with goblet cells. The trichilemmal cysts exhibited stratified squamous epithelium without a granular cell layer (trichilemmal keratinization) and contained keratin in the lumen. The dermoid cyst consisted of stratified squamous epithelium where sebaceous glands and hair follicles were intermixed. The apocrine hidrocystoma had several cystic spaces lined by a few layers of columnar epithelium with eosinophilic cytoplasm and exhibited secretion of amorphous mound materials into the lumen, which is consistent with decapitation secretion. The lesion classified as a lacrimal gland cyst had enlarged ducts made up of a two-layer epithelium, which possibly arose from the accessory lacrimal gland in the eyelid conjunctiva.

Unclassifiable cysts. A total of 6 cases were histologically unclassifiable cysts. One cyst resembled an apocrine hidrocystoma, and the other an IKC; however, they had atypical features. At initial presentation, the origin of the lesion was clinically undetermined following resection of the cystic lesion. These four cases lacked epithelia and/or contents, but presumed diagnoses were eccrine hidrocystoma or epidermal cyst. The following two cases are the unclassifiable cysts that were suspected as apocrine hidrocystoma and IKC, respectively.

Suspected case of apocrine hidrocystoma. An 86-year-old male presented with a brownish colored translucent nodule in the right upper eyelid margin (Fig. 3A). Histologically, the cyst was made up of multiple layered epithelia. Several epithelial cells presented decapitation secretion. The content of the cyst was an eosinophilic amorphous structure with a cholesterol cleft as well as granuloma infiltrated by foamy cells and multinucleated giant cells (Fig. 3B and C). The cyst resembled an apocrine hidrocystoma; however, the cyst contents, as observed in this case, were not described in the previous report (4). Therefore a definite histological diagnosis could not be made.

Suspected case of IKC. A 49-year-old male presented with a brownish colored nodule beneath the palpebral conjunctiva in the right upper eyelid (Fig. 4A). Histologically, the wall of the cyst was stratified squamous epithelium without a granular layer, though included pigment granules (Fig. 4B and C). These granules were stained by Fontana-Masson staining, which was consistent with melanin. The content of the cyst was keratin together with foreign-body inflammatory granulomatous tissue due to rupture of the cyst (Fig. 4D). Although the cyst could have been considered a type of IKC, melanin within the cyst wall has not been described in the previous reports $(1,2,6,7)$. Therefore, the histological findings did not enable a definitive diagnosis.

\section{Discussion}

Primarily, the current study determined that the upper eyelid was the most frequent site of the cystic lesions in the studied patients. Although the reason remains unknown, the longitudinal length of eyelid skin and tarsus being longer in the upper eyelid than the lower eyelid, and the number of meibomian glands and hair follicles being anatomically greater in the upper eyelid than the lower eyelid may be causes. These known facts mean that the upper eyelid possess a greater volume and 
Table I. Diagnoses and clinical profiles of the 35 cystic lesions in the present study.

\begin{tabular}{|c|c|c|c|c|c|}
\hline Origin and diagnosis & No. $(\%)$ & Age & Sex & Laterality & Location \\
\hline \multicolumn{6}{|l|}{ Eyelid skin and its appendages } \\
\hline \multirow[t]{16}{*}{ Epidermal cyst } & $16(46)$ & 77 & $\mathrm{~F}$ & $\mathrm{R}$ & Upper eyelid \\
\hline & & 67 & $\mathrm{~F}$ & $\mathrm{~L}$ & Upper eyelid \\
\hline & & 60 & M & $\mathrm{L}$ & Medial canthus \\
\hline & & 74 & M & $\mathrm{L}$ & Medial canthus \\
\hline & & 78 & $\mathrm{~F}$ & $\mathrm{R}$ & Upper eyelid \\
\hline & & 5 & $\mathrm{~F}$ & $\mathrm{R}$ & Upper eyelid \\
\hline & & 87 & $\mathrm{~F}$ & $\mathrm{~L}$ & Upper eyelid \\
\hline & & 31 & M & $\mathrm{R}$ & Upper eyelid \\
\hline & & 28 & $\mathrm{~F}$ & $\mathrm{R}$ & Upper eyelid \\
\hline & & 55 & M & $\mathrm{R}$ & Lower eyelid \\
\hline & & 70 & $\mathrm{~F}$ & Unknown & Unknown \\
\hline & & 23 & $\mathrm{~F}$ & $\mathrm{~L}$ & Medial canthus \\
\hline & & 77 & $\mathrm{~F}$ & $\mathrm{~L}$ & Upper eyelid \\
\hline & & 66 & $\mathrm{~F}$ & $\mathrm{R}$ & Lower eyelid \\
\hline & & 78 & $\mathrm{~F}$ & $\mathrm{R}$ & Medial canthus \\
\hline & & 3 & $\mathrm{~F}$ & $\mathrm{~L}$ & Upper eyelid \\
\hline \multirow[t]{3}{*}{ Trichilemmal cyst } & $2(6)$ & & & & \\
\hline & & 76 & M & $\mathrm{R}$ & Upper eyelid \\
\hline & & 70 & $\mathrm{M}$ & $\mathrm{L}$ & Upper eyelid \\
\hline Dermoid cyst & $1(3)$ & 15 & M & $\mathrm{R}$ & Upper eyelid \\
\hline Apocrine gland cyst & $1(3)$ & 11 & M & $\mathrm{R}$ & Medial canthus \\
\hline \multicolumn{6}{|l|}{ Tarsus } \\
\hline \multirow[t]{6}{*}{ Intratarsal keratinous cyst } & $5(14)$ & & & & \\
\hline & & 83 & $\mathrm{~F}$ & $\mathrm{R}$ & Upper eyelid \\
\hline & & 76 & M & $\mathrm{R}$ & Upper eyelid \\
\hline & & 74 & M & $\mathrm{L}$ & Upper eyelid \\
\hline & & 61 & $\mathrm{~F}$ & $\mathrm{R}$ & Upper eyelid \\
\hline & & 48 & $\mathrm{~F}$ & $\mathrm{R}$ & Upper eyelid \\
\hline \multicolumn{6}{|l|}{ Palpebral conjunctiva } \\
\hline \multirow[t]{4}{*}{ Conjunctival cyst } & $3(9)$ & & & & \\
\hline & & 80 & $\mathrm{~F}$ & $\mathrm{~L}$ & Lower eyelid \\
\hline & & 62 & $\mathrm{M}$ & $\mathrm{L}$ & Upper eyelid \\
\hline & & 47 & $\mathrm{~F}$ & $\mathrm{~L}$ & Upper eyelid \\
\hline Lacrimal gland cyst & $1(3)$ & 54 & M & $\mathrm{L}$ & Upper eyelid \\
\hline \multirow[t]{7}{*}{ Unclassified } & $6(17)$ & & & & \\
\hline & & 24 & M & $\mathrm{R}$ & Lower eyelid \\
\hline & & 70 & $\mathrm{M}$ & $\mathrm{L}$ & Lower eyelid \\
\hline & & 86 & M & $\mathrm{R}$ & Upper eyelid \\
\hline & & 49 & M & $\mathrm{R}$ & Upper eyelid \\
\hline & & 55 & $\mathrm{~F}$ & Unknown & Unknown \\
\hline & & 65 & $\mathrm{~F}$ & $\mathrm{R}$ & Unknown \\
\hline
\end{tabular}

F, female; M, male; R, right; L, left.

area, thus resulting in a greater number of cystic lesions in the upper eyelid than the lower eyelid.

Deprez and Uffer (3) reported that among 5,504 eyelid skin tumors excluding chalazion and tumors of the palpebral conjunctiva, benign tumors made up $84 \%$ of the cases, and the frequency of the subtypes was squamous cell papilloma (23\% in benign tumors), seborrheic keratosis (19\%), melanocytic nevus (18\%), epidermal cyst (11\%) and hidrocystoma (7\%). Out of 849 cystic lesions, the most frequent subtypes were epidermal cyst (58\% in cystic lesions), followed by hidrocystoma (38\%) 

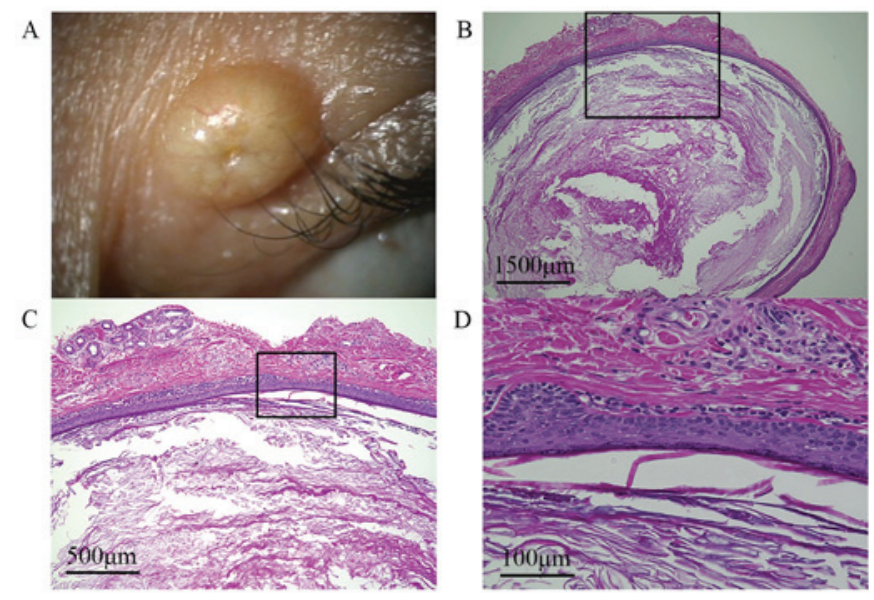

Figure 1. A representative case of an epidermal cyst. (A) A 67-year-old male presented with a subcutaneous nodule with a central punctum in the left upper eyelid. (B) Histologically, the nodule was a single cyst (hematoxylin and eosin; scale bar, 1,500 $\mu \mathrm{m}$ ). (C) Magnification of the rectangular region in (B) showed that the wall of the cyst consisted of normal stratified squamous epithelium with a granular layer (hematoxylin and eosin; scale bar, $500 \mu \mathrm{m}$ ). (D) The content of the cyst was laminated layers of keratin (hematoxylin and eosin; scale bar, $100 \mu \mathrm{m})$.
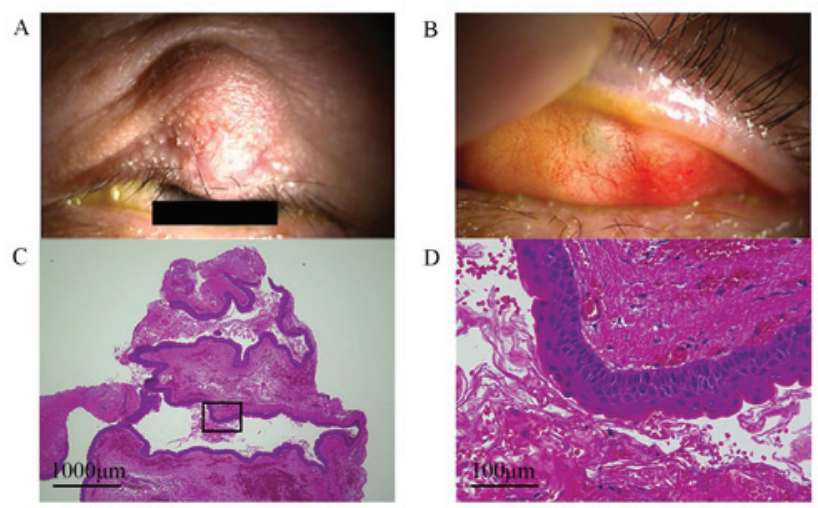

Figure 2. A representative case of an intratarsal keratinous cyst. (A) A 74-year-old male presented with an eyelid nodule. (B) The everted eyelid of the patient revealed a smooth, white, noninflamed lesion, indicating that the lesion was located within the tarsal plate. (C) Histologically, the lesion was a ruptured cyst (hematoxylin and eosin; scale bar, 1,000 $\mu \mathrm{m}$ ). (D) Magnification of the rectangular region in (C) showed that the cyst wall contained undulating stratified squamous epithelium without a granular layer and lacked inflammation. The content of the cyst was string-like keratin (hematoxylin and eosin; scale bar, $100 \mu \mathrm{m}$ ).

and dermoid cyst (4\%) (3). Epidermal cysts typically form a smooth dome-shaped elevation of varying size that may have an opening site to the eyelid surface (4). Epidermal cysts histologically have their wall constituted by keratinized stratified squamous epithelium with a granular layer, in which the content is laminated layers of keratin (4). In the current study, epidermal cysts were the most common lesions in the eyelid cystic lesions as reported previously (3).

Interestingly, IKCs were the second most common lesion in our cohort. IKC is characterized by localization of the cystic lesion in the tarsus. Clinically, an IKC presents as a lump under the eyelid skin or beneath the conjunctiva that is stationary and does not move with manipulation of the overlying eyelid skin $(1,6)$. Histologically, the cyst contains an undulating
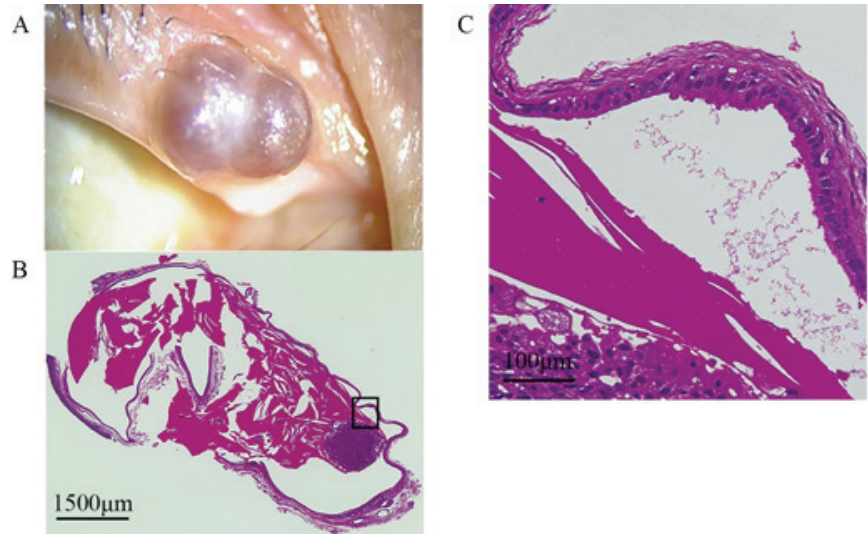

Figure 3. Suspected case of apocrine hidrocystoma. (A) An 86-year-old male presented with a brownish colored translucent nodule in the right upper eyelid margin. (B) Histologically, the lesion was a single cyst (hematoxylin and eosin; scale bar, 1,500 $\mu \mathrm{m}$ ). (C) Magnification of the rectangular region in (B) showed that the cyst was made up of multiple layered epithelia with myoepithelial cells. The epithelium presented with decapitation secretion. The content of the cyst was eosinophilic amorphous structure with a cholesterol cleft as well as granuloma infiltrated by foamy cells and multinucleated giant cells (hematoxylin and eosin; scale bar, $100 \mu \mathrm{m}$ ).
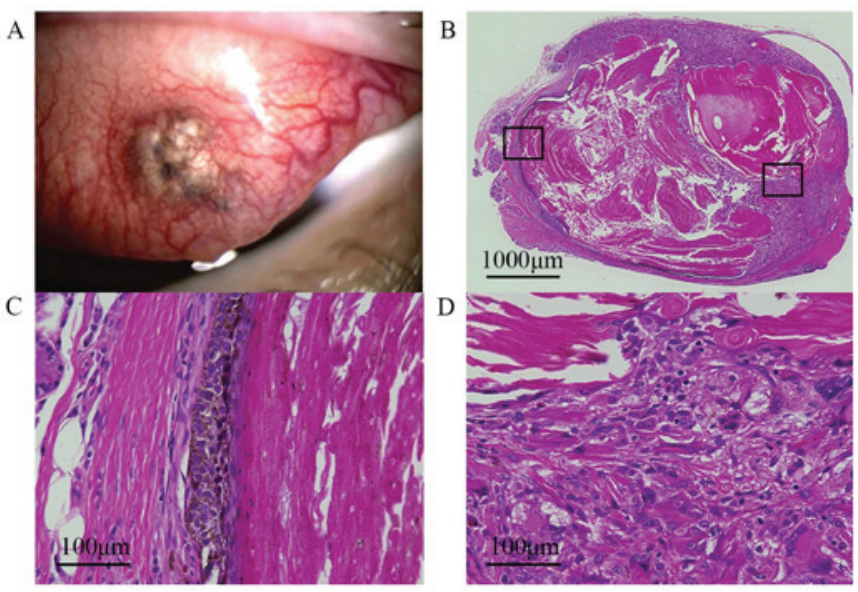

Figure 4. Suspected case of IKC. (A) A 49-year-old male presented with a brownish colored nodule beneath the palpebral conjunctiva in the right upper eyelid. (B) Histologically, the lesion was an inflamed ruptured cyst (hematoxylin and eosin; scale bar, 1,000 $\mu \mathrm{m}$ ). (C) Magnification of the left rectangular region in (B) showed that the wall of the cyst was a stratified squamous epithelium, notably including pigment granules, without a granular layer (hematoxylin and eosin; scale bar, $100 \mu \mathrm{m}$ ). (D) Magnification of the right rectangular region in (B) showed that the content of the cyst was keratin together with foreign-body inflammatory granulomatous tissue (hematoxylin and eosin; scale bar, $100 \mu \mathrm{m})$.

surface lined by 4-5 layers of polygonal keratinocytes that exhibit abrupt keratinization without keratohyalin granules (trichilemmal keratinization) (1,7). The lumen of the cysts is filled with abundant, eosinophilic, refractile and string-like keratin (7). By contrast, neither goblet cells nor sebaceous cells are intermixed in the wall of IKC (1). As IKCs are among the more recent cysts to be characterized (1), and are likely to mimic the histological findings of epidermal cysts, IKCs may have been classified as epidermal cysts in previous years. Indeed, IKCs have been reported as the third most common lesions following chalazia and sebaceous tumors among the lesions originating from the tarsal plates (1). Collectively, the 
present findings indicated that IKCs were also a fairly common histological subtype among cystic lesions of the eyelid in the test population.

Previous studies reported that sporadic IKCs presented with a single nodule (95\%), and could occur in the upper eyelids (95\%), were slightly more frequent in men (56\%) and were more frequent in older individuals (mean age, 56 years) (6). By contrast, eyelid cysts, typically IKCs, tended to occur bilaterally as multiple eyelid cysts (80\%) and were observed at earlier ages (mean age, 40 years) in Gorlin (nevoid basal cell carcinoma) syndrome (6). In the current study, all of the IKCs were sporadic, and the mean age was $68 \pm 14$ years. All of these lesions involved the upper eyelid as a single cyst, and there was no obvious gender predilection (two males and three females).

In the current study, 6 cysts were not classified. Of these, 4 out of 6 cases were unclassifiable because they lacked epithelium and/or the contents of the cysts, possibly due to dropout during the process of obtaining pathological sections. The other 2 cases were initially considered an apocrine hidrocystoma and an IKC. However, the former was atypical as an apocrine hidrocystoma since the cyst exhibited an eosinophilic amorphous structure with cholesterol cleft in the lumen, which is not commonly found in an apocrine hidrocystoma (4). The latter was morphologically atypical as an IKC because the epithelium exhibited melanin. Jakobiec et al (1) reported that immunostaining of cytokeratin (CK)17, carcinoembryonic antigen (CEA) and epithelial membrane antigen (EMA) may distinguish IKCs from epidermal cysts; CK17, CEA and EMA are positive for IKCs, and negative for epidermal cysts. Further immunopathological studies would have been required to elucidate the origin of the cystic lesions.

There were limitations to the current study. Firstly, the number of patients with cystic lesions was relatively small due to it being a single-center study. Therefore, the study did not include other eyelid cystic lesions such as eccrine hidrocystoma, milia and steatocystoma that were not encountered in our center. Secondly, because it was a retrospective study, the follow-up periods, and the amount of information obtained from the medical records differed for each patient. Therefore, the location of cysts in certain patients was unknown, and some cysts may have recurred after the follow-up period.

In conclusion, the upper eyelid was the most frequent site of cystic lesions in the eyelid in the studied cohort. The most common diagnoses were epidermal cysts followed by IKCs, though unclassifiable cysts were intermixed with the total lesions studied. The current study indicates that the incidence and differential diagnosis of eyelid cystic lesions may contribute to the implementation of appropriate treatment for patients with eyelid tumors.

\section{Acknowledgements}

Not applicable.

\section{Funding}

No funding was received.

\section{Availability of data and materials}

The datasets used and/or analyzed during the current study are available from the corresponding author on reasonable request.

\section{Authors' contributions}

YS drafted the manuscript. HKO collected the patient data. YS and KI analyzed and interpreted patient data. SK and HKO performed histological classification. SK, and SI critically revised the manuscript. All authors read and approved the final manuscript.

\section{Ethics approval and consent to participate}

The IRB for clinical research of Hokkaido University Hospital approved the current study (IRB approval no. 016-0426). The research adhered to the tenets of the Declaration of Helsinki.

\section{Patient consent for publication}

Written informed consent or opt out for approval for the publication of patient information was obtained.

\section{Competing interests}

The authors declare that they have no competing interests.

\section{References}

1. Jakobiec FA, Mehta M, Iwamoto M, Hatton MP, Thakker M and Fay A: Intratarsal keratinous cysts of the Meibomian gland: Distinctive clinicopathologic and immunohistochemical features in 6 cases. Am J Ophthalmol 149: 82-94, 2010.

2. Lucarelli MJ, Ahn HB, Kulkarni AD and Kahana A: Intratarsal epidermal inclusion cyst. Ophthal Plast Reconstr Surg 24: 357-359, 2008.

3. Deprez M and Uffer S: Clinicopathological features of eyelid skin tumors. A retrospective study of 5504 cases and review of literature. Am J Dermatopathol 31: 256-262, 2009.

4. Pe'er J: Pathology of eyelid tumors. Indian J Ophthalmol 64: 177-190, 2016.

5. Zürcher M, Hintschich CR, Garner A, Bunce C and Collin JR: Sebaceous carcinoma of the eyelid: A clinicopathological study. Br J Ophthalmol 82: 1049-1055, 1998.

6. Wolkow N, Jakobiec FA and Yoon MK: Intratarsal keratinous eyelid cysts in Gorlin syndrome: A review and reappraisal. Surv Ophthalmol 63: 711-718, 2018.

7. Patel VS, Meyer DR and Carlson JA: Intratarsal keratinous cysts of the meibomian gland (a sebaceous duct cyst): Report of 2 cases. Am J Dermatopathol 33: 624-627, 2011. 